\title{
La confidencialidad de la consulta psiquiátrica y el deber de protección a terceros: El caso Tarasoff Tarasoff's case
}

Rodrigo A. Salinas R. ${ }^{1}$

\section{Introducción}

$\mathrm{E}$ I neologismo que da el nombre a esta sección, N euroética, es de reciente creación. De acuerdo a la información contenida en el sitio web que la Universidad de Pennsylvania ha creado para abordar esta materia (http://neuroethics. upenn.edu), la N euroética se debe entender como "la ética de la neurociencia", del mismo modo como el término bioética define a la ética de las ciencias biomédicas, de un modo más general. Las materias que aborda dicen relación con la inagotable fuente de dilemas éticos que han surgido con el desarrollo de la tecnología asociada a esta disciplina, así como las perspectivas futuras de desarrollo que las ciencias básicas prometen para las próximas décadas. Como a menudo ocurre con las palabras cuando éstas son creadas, en el proceso de encontrar su centro van cambiando de sentido y contenido, casi imperceptiblemente. A la definición original del término, utilizada -entre otros- por el periodista norteamericano William Safire ${ }^{1}$, que definía a la Neuroética como "el campo de la filosofía que delibera sobre lo correcto y lo incorrecto en el tratamiento o en la mejora del cerebro humano" se fueron sumando otras, entre ellas la del distinguido investigador de la conducta, Dr. M ichael Gazzaniga, para quien esta nueva disciplina va mucho más allá, comprendiendo el examen de "cómo nos quere- mos relacionar con los aspectos sociales de la enfermedad, la normalidad, la mortalidad, los estilos de vida y la filosofía de lo viviente, a partir de la comprensión de sus mecanismos cerebrales subyacentes". El interés llevó, incluso, a la Comisión Presidencial de Bioética del Gobierno de Estados Unidos, a dedicar un área especial de discusión a esta materia, con sesiones dedicadas específicamente a abordarla y abundante información que está disponible en su sitio web (http://www. bioethics.gov). El inicio del nuevo milenio fue marcado por una serie de simposios y reuniones que debatieron extensamente sobre esta nueva disciplina. Muchos estimaron que el nombre era poco feliz, o que podía resultar excluyente para otros profesionales, pero lo que parecía quedar claro de la discusión, según uno de los co-organizadores de una de estas conferencias, era que "podemos Ilamarlo como queramos, pero el tren de la Neuroética ya dejó la estación". Como no nos gusta que nos deje el tren -a nadie le gustahemos dado origen a esta sección con el ambicioso propósito de dar cabida a dilemas surgidos de las más diversas vertientes de nuestras disciplinas profesionales. Desde aquellos que se relacionan con la confidencialidad de la información entregada por los pacientes en la consulta psiquiátrica, hasta los que dicen relación con el impacto sobre la sociedad que puede resultar de las nuevas técnicas de intervención farmacológica sobre la mente. El formato de esta sección consta de

1 Editor. Revista Chilena de Neuro-Psiquiatría. 
una viñeta que es comentada por dos o más expertos en la materia, desde la perspectiva legal, ética, 0 de nuestras profesiones ligadas a las neurociencias, clínicas o básicas. En este número le damos inicio, recurriendo a uno de los casos más clásicos de la discusión ética en torno a la atención psiquiátrica, el caso de Tatiana Tarasoff, que se relata (y comenta) a continuación.

\section{La muerte de Tatiana Tarasoff ${ }^{2}$}

Prosenjit Poddar, nacido en India, llegó a estudiar a California a fines de los sesenta. En ese estado conoció, en el otoño de 1968, a Tatiana Tarasoff, durante actividades recreativas de la residencia estudiantil donde vivía. Iniciaron una relación que Prosenjit interpretó como seria, visión que no era compartida por Tatiana. Al darse cuenta Tatiana de la disparidad de opiniones le dijo a Prosenjit que estaba comprometida con otro hombre y que no deseaba iniciar una relación con él. El rechazo provocó en el estudiante una seria crisis emocional; descuidó su apariencia personal y su ánimo se tornó fuertemente depresivo.

Durante 1969, con posterioridad a un viaje de Tatiana a Brasil, Prosenjit buscó apoyo profesional y en agosto de ese año figuraba como paciente externo en el Cowell Memorial Hospital. En un comienzo fue visto por el Dr. Stuart Gold, psiquiatra, para posteriormente ser derivado al cuidado del psicólogo Lawrence Moore. En su novena sesión con M oore, el 18 de agosto de 1969, el estudiante le confidenció que iba a matar a una mujer, claramente identificable como Tatiana, cuando ésta retornase de Brasil. Dos días después, M oore notificó a la policía que Poddar sufría de una reacción esquizofrénica paranoide y que se encontraba en riesgo de dañarse a si mismo, o de dañar a otros. La policía del campus retuvo a Poddar y lo liberó una vez satisfecha que el estudiante había cambiado de actitud y, aparentemente, por la intercesión del Director de Psiquiatría del centro asistencial, quien solicitó al Jefe de Policía la devolución de la carta de Moore y or- denó a este último la destrucción de sus registros de la terapia, así como las copias de la carta enviada a la policía. En octubre siguiente Tatiana, al regresar de Brasil, continuó siendo acosada por Poddar, siendo acuchillada y muerta por éste el 29 de ese mes, al negarse a sus requerimientos.

Moore, en su testimonio judicial, reveló que Poddar había dado a conocer sus intenciones de matar a Tatiana durante las sesiones de terapia. Subsecuentemente, durante las audiencias, surgieron serias discrepancias entre los peritos presentados por las partes respecto al exacto diagnóstico del imputado, terminando en una condena de primera instancia por homicidio en segundo grado. Los recursos presentados a la Corte de Apelaciones $y$, posteriormente, a la Corte Suprema de California, sin embargo, terminaron por encontrar vicios de procedimiento que obligaban a reconducir el juicio, lo que fue conmutado por la expulsión de Poddar a la India donde, de acuerdo algunos autores, se encuentra felizmente casado con una abogada.

Los padres de Tatiana Tarasoff, por su parte, decidieron querellarse en contra de la Universidad de California y sus psicoterapeutas. Una de la quejas contenidas en la demanda era que los psicoterapeutas no habían dado oportuno aviso a Tatiana, 0 a sus cercanos, de las intenciones que tenía Prosenjit de matar a su pretendida. Tanto la Corte del Condado de Alameda, como la Corte de Apelaciones correspondiente, encontraron que no había razones para condenar, agregando esta última que al no existir relación alguna entre el psicoterapeuta y Tatiana, o sus padres, no existía, por consiguiente, la obligación de informar. La Corte Suprema de California, sin embargo, pensó distinto. En su decisión final sostuvo, entre otras consideraciones, la siguiente: “... los terapeutas no pueden escapar a su responsabilidad simplemente porque Tatiana no era su paciente. Cuando un terapeuta determina, o de acuerdo a los estándares de su profesión, debiese determinar, que su paciente representa un serio peligro para otros, le asiste la obligación de ejercer cuidados razonables dirigidos a proteger a las victimas de tal peligro. El cumplimiento de 
este deber puede requerir del terapeuta tomar una o más medidas, dependiendo de la naturaleza del caso. Así, puede llevarlo a advertir a la posible víctima o a otros que puedan advertir a la víctima del peligro, notificar a la policía o tomar las medidas que sean razonablemente necesarias en esas circunstancias".

\section{Referencias}

1. Farah M. Emerging ethical issues in neuroscience. Nature N euroscience 2002; 5: 1123-9.

2. Buckner $F$, Firestone $M$. Where the Public Peril Begins: 25 Years after Tarasoff. J Leg M ed 2000; 21: 187-222.

\section{Comentarios sobre el caso Tarasoff Dr. Carlos Téllez T.}

Clínica Psiquiátrica U niversitaria, Universidad de Chile

El caso Tarasoff nos plantea problemas éticos, legales y prácticos. Los primeros tienen que ver con el quiebre de la confidencialidad en relación a un potencial daño grave a un tercero, el Código de Ética del Colegio M édico (art 38 letra d) explicita que es lícita la ruptura del secreto profesional en casos donde hay amenazas o potencial riesgo real de daño físico a otras personas. A su vez el Código Sanitario en los arts. 20 y 40 establece la obligatoriedad de denuncia de enfermedades trasmisibles y venéreas.

Los problemas legales que derivan de los casos "tipo Tarasoff" tienen que ver con las demandas civiles que pudiesen interponer las víctimas 0 sus familias cuando un tercero es dañado por un paciente y su terapeuta, estando al tanto del riesgo, optó por callar cuidando el secreto profesional. En el Código Procesal Penal no existen disposiciones de denunciar un delito que aún no se comete.

Quedan pocas dudas respecto a que el quiebre del secreto profesional es ético ante el riesgo de grave daño a tercero. Sin embargo, a través de tres situaciones clínicas reales que podríamos de nominar "tipo Tarasoff" podremos visualizar la complejidad del tema y la necesidad de revisar cuidadosamente cada caso en particular.
1. Chofer de bus interprovincial que consulta por depresión, cuenta durante la entrevista que mientras trabaja producto de la desesperanza y angustia que siente ha pensado tirar el bus por un barranco.

Afortunadamente el paciente aceptó voluntariamente hospitalizarse, de no haberlo hecho teníamos la posibilidad de una hospitalización no voluntaria de urgencia o administrativa. En el sistema privado es más difícil proceder a este tipo de hospitalización si no hay recursos económicos. En este caso hubiese sido aceptable, después de conversarlo con el paciente, informar a familiar de los riesgos existentes y obligar a licencia manteniendo estrictos controles médicos. De no haber familiares, después de conversar con el paciente, habría que haber llamado a su empresa y hacer ver que el paciente no se encuentra en condiciones de trabajar hasta tener una autorización del tratante, esto cuidando de entregar el mínimo de información suficiente a un superior responsable.

2. Paciente hospitalizado por un trastorno delirante celotípico que explicita su idea de matar a su cónyuge en medio de crisis paranoide.

Este caso, obliga a tomar todas las medidas para proteger a la cónyuge, informándola directamente del problema y de ser necesario avisar a la policía. Debe procederse a hospitalización ad- 
ministrativa explicitando en el informe a la autoridad sanitaria de los riesgos a terceros existentes. En esta situación, dado que se trata de un cuadro psicótico que con el debido tratamiento debería devolver el juicio de realidad a nuestro paciente, éste eventualmente incluso debería agradecer el haberlo protegido a él y su esposa durante la crisis.

3. Paciente con una parafilia sádica y trastorno de personalidad grave que pide ayuda ya que lo atormentan fantasías recurrentes de torturar y matar a mujeres desconocidas, ha iniciado recientemente conductas sádicas (golpes y humillación) con prostitutas, las que habrían sufrido lesiones hasta de mediana gravedad. Estas prácticas hasta ahora se han limitado a prostitutas, su esposa no ha notado nada fuera de lo común en el paciente.

Este caso, el más complejo, nos impone varias dificultades. Primero, tenemos un individuo que con bastante seguridad dañará gravemente a alguna mujer, Segundo, no tenemos a quien advertir ya que son miles las potenciales víctimas. Tercero, la policía o fiscales lo podrían detener pero sería inútil ya que seguramente negaría lo reportado a nosotros y no habiendo denuncias tendrán que dejarlo en libertad. Cuarto, una hospitalización no voluntaria no ayudaría ya que el paciente dejaría de cooperar y el tratamiento que necesariamente debe ser de muy largo plazo no se cumpliría. Quizás lo recomendable sería tomar positivamente que haya sido el paciente quien consulta, incentivar la confianza en el/la terapeuta e intentar terapia psicológica y farmacológica destinada a mantener su problemática a nivel de fantasías y bajar niveles de impulsividad y libido. Con la autorización del paciente conversar con su pareja e intentar control externo. Este caso pone de relieve el hecho que no siempre en estas situaciones hay que quebrar el secreto profesional ya que por una parte no es efectivo denunciar y por otro el quebrar el secreto hace casi invariablemente que estos pacientes pierdan la confianza y no vuelvan a consultar, con lo que lejos de ayudar incrementa el riesgo de violencia.

A modo de cierre se podría decir que en caso de enfrentar situaciones "tipo Tarasoff" se recomienda1,2:

1. Hacer una buena evaluación clínica tratando de dimensionar el riesgo real, conversar abiertamente primero con el paciente de los riesgos y de la eventual obligatoriedad del médico de hacer la denuncia, esto a veces tiene valor terapéutico. Debe evitarse que el paciente se vea sorprendido con nuestra denuncia.

2. Evaluar muy bien quien será la persona o institución que reciba la denuncia, esto dependerá del tipo de violencia y su gravedad. Considerar siempre que la información entregada debe ser la mínima suficiente para prevenir el daño.

3. Los casos son siempre distintos por lo por lo que no hay fórmulas generales.

4. Siempre discutir estos casos con colegas, idealmente de más experiencia.

5. Dejar registro en la ficha clínica de los hallazgos psicopatológicos, de la problemática, de las decisiones tomadas y su argumentación. En caso de haber discutido el caso hacer resumen de las conclusiones.

\section{Referencias}

1. Walcott D M, Cerundolo P, Beck J C. Current analysis of the Tarasoff duty: an evolution towards the limitation of the duty to protect. Behav Sci Law 2001; 19 (3): 325-43.

2. Gutheil T G. Moral justification for Tarasoff-type warnings and breach of confidentiality: a clinician's perspective. Behav Sci Law 2001; 19 (3): 345-53. 


\section{Los variados rostros de la confidencialidad: Legalidad y eticidad Dr. Gustavo Figueroa C.}

Editor Asociado Psiquiatría

Revista Chilena de N euro-Psiquiatría

El caso de Tatiana Tarasoff y Prosenjit Poddar se ventiló en los tribunales durante los años que se estaba gestando la bioética americana y produjo un impacto en la National Commission, convocada por el Congreso de los Estados Unidos, que redactó el Informe Belmont (1978). Este es el motivo por el cual analizar bioéticamente el caso es partir de la sentencia legal como dato primero, como si ésta tuviera prioridad en el orden de la moralidad y, proceder en acuerdo a la bioética, es un momento posterior, que se limita a admitir, modificar o impugnar los argumentos judiciales. Este sesgo legalista es inherente a la bioética americana, y esta confusión o prejuicio ha determinado a la del resto de Occidente, aún en contra de su tradición hipocrática de "aquello que jamás deba trascender, lo callaré, teniéndolo por secreto". Contrariamente, la American Medical Association señala rotundamente que el médico "deberá salvaguardar las confidencias del paciente dentro de los límites que establezca la ley".

De las muchas caras inherentes a la confidencialidad queremos reducirnos a tres: ¿cuánto sabía Lawrence M oore de Poddar?, ¿por qué notificó a la policía del campus para que lo arrestaran? y ¿debió alertar a Tatiana Tarasoff? Respecto al conocimiento que tenía de Poddar, éste había sido evaluado por el psiquiatra Stuart Gold antes de enviarlo a psicoterapia. La ficha clínica (y/o la discusión del caso antes de iniciar el tratamiento) es el material indispensable que debe estar a disposición del psicoterapeuta. Parece claro que la información completa debió estar en manos de Lawrence M oore, en toda su profundidad y sin existir limitación alguna; aún más, fue deber suyo el haberla recabado para ejecutar mejor su asistencia profesional. El acceder a la historia como soporte documentado es decididamente restringido, salvo al psicoterapeuta y su equipo ¿No sucedió así? ¿O fue un error de diag- nóstico que pasó por alto el (probable) cuadro psicótico y éste sólo se hizo patente durante la psicoterapia? ¿0 se postuló efectuar una psicoterapia ambulatoria a pesar de la posibilidad de que Poddar sufriera de una "reacción esquizofrénica" por los beneficios que podría alcanzar?

El siguiente dilema, avisar a la policía, encierra dos asuntos distintos. Primero, efectuar una interconsulta al psiquiatra y su supervisor para decidir los pasos a seguir. Moore efectivamente acordó, siguiendo a los doctores Gold y Yandell, solicitar la reclusión para observar la conducta de Poddar. Como en la situación anterior, parece claro que fue éticamente pertinente revelar información a los psiquiatras máxime que por lo menos uno ya lo conocía con anterioridad, y además Poddar estaba al corriente de las reglas usuales del policlínico universitario concernientes al intercambio de opiniones confidenciales entre profesionales para todos los pacientes bajo tratamiento en éste. ¿Debieron reevaluarlo previamente, en conjunto con el resto del equipo? Aunque ésta es una decisión preponderantemente técnica, por iguales motivos también era éticamente justificado discutir su historial completo con la totalidad del grupo de salud mental. Aún más, debido a la gravedad del riesgo involucrado era moralmente justificado exponer en detalle el desarrollo del proceso psicoterapéutico a ambos supervisores, en busca de indicios premonitorios de su idea homicida y de posibles errores o problemas contratransferenciales en la conducción del tratamiento. Segundo, dar a conocer el diagnóstico psiquiátrico de Podddar, oralmente primero (a los agentes Atkinson y Teel) y luego por escrito al Jefe de Policía William Beall, resulta altamente controvertible desde la perspectiva bioética. ¿Era esta revelación absolutamente indispensable para justificar ante la ley su potencial peligrosidad y, por tanto, su necesidad de reclusión? El diagnóstico médico es privado, producto de la interacción que se ha llevado a cabo en la intimidad de las sesiones y, salvo que exista una causa poderosísima, le pertenece al paciente y su médico (o equipo). Lo que interesaba eran los pensamientos y conductas objetivas que ha- 
cían aconsejable su internación, y no su etiqueta diagnóstica. Su mal uso, en parte por falta de conocimientos técnicos o su mala comprensión, fue claro en el caso Poddar puesto que fueron finalmente los agentes los que determinaron si tenía o no una psicosis esquizofrénica, en sus palabras legales, si contaba con la racionalidad como para seguir haciendo su vida corriente. En otros términos, se traspasaron los límites de la confidencialidad ética, se ignoraron (quizás) reglas técnicas con alcances morales y se restringieron a lo judicial (mal aplicado), dándole una primacía injustificada.

Por último, el no poner sobre aviso a Tatiana Tarasoff supuso un fallo al no acudir a una ética de naturaleza consecuencialista puesto que Poddar representaba un peligro para ella. Es no haber hecho prevalecer al principio de justicia por sobre los otros tres (autonomía, no-maleficencia, beneficencia), principio que a su vez justificaría no respetar la confidencialidad. Como siempre, los principios no son absolutos sino prima facie, esto es, valen mientras no existan circunstancias especiales que determinen que otros sean superiores. De ahí que se puede acudir a distintos conceptos sustantivos en ética como para poder dirimir mejor la encrucijada. En el caso de Poddar, uno puede recurrir a evaluar si la medida de advertir obedece no al par ordinario/extraordinario (como sucede en pacientes en coma permanente y se plantea la posibilidad de reanimación a continuación de un paro cardíaco), sino a la polaridad proporcionado/desproporcionado ¿era proporcionado o desproporcionado alertar a Tatiana como para explicar quebrar la confidencialidad? Admitiendo que se desconocen las circunstancias clínicas precisas como para ponderar bien su peligrosidad ( presencia de ocurrencias aisladas o ideas delirantes inconmovibles, historial de conductas agresivas, etc), parece desproporcionada considerando que hasta esos instantes no se había pensado jamás en una esquizofrenia "severa" ni en otro trastorno psicótico o de personalidad "graves". Además, ¿qué desequilibrio podía provocar en Tatiana? ¿Qué se le iba a ofrecer psiquiátricamente como com- pensación en caso de error? ¿0 quedaría en manos de la justicia velar por ella a continuación? En cualquier caso, era desproporcionada sin haber aplicado con determinación y firmeza las medidas anteriores, reevaluación rigurosa por equipo psiquiátrico, análisis del proceso psicoterapéutico y/o hospitalización para observación estricta. El trágico desenlace no justifica lo desproporcionado, sino nos recuerda más bien lo azaroso o imprevisible de nuestra profesión.

Algunas enseñanzas se desprenden del caso Poddar-Tarasoff.:

1. La confidencialidad ha ido perdiendo vigencia y fuerza en los últimos años producto del giro legalista que ha inundado a la medicina, que confunde y subordina la ética al derecho, lo bueno o malo con lo legal o ilegal;

2. Las revelaciones del paciente poseen un carácter de "información sensible" que es consecuencia de pertenecer a la intimidad y vida privada del sujeto, por lo que necesitan de una protección o blindaje especial por parte de los profesionales;

3. La psicoterapia resulta substancialmente delicada porque, a propósito, se suele entrometer y escarbar en lo más humano y oculto del ser, sus deseos, fantasmas, esperanzas, ambiciones, perversiones, envidias, obsesiones, por lo que no respetar su estructura especial y única de "confidencia" puede conducir no sólo a desvirtuarla sino a exterminarla $y$, dentro de poco, dejar de pertenecer al conjunto de herramientas terapéuticas a disposición del médico salvo como simple apoyo empático;

4. Las excepciones que necesariamente deben limitarla requieren ser estudiadas nuevamente, habida cuenta los avances técnicos arrolladores que permiten adueñarse de partes decisivas de la interioridad del enfermo, y el clima histórico de auto-revelación sin límites que rodea, impregna y contamina a la medicina del siglo XXI;

5. Se debe de reconocer que "la confidencialidad en medicina es un concepto decrépito", como se asegura desde hace al gunos años, porque es ampliamente ignorada y violada de manera 
sistemática en la atención médica rutinaria, como hospitales, consultorios, empresas aseguradoras, organismos estatales fiscalizadores, industria de la comunicación, aún en la vida social de médicos y psicólogos que comentan y discuten sobre sus pacientes sin consideración ninguna por el valor que supone el respeto por la interioridad. Resulta ostensible que la declaración de la Asociación M édica M undial no fue sino un anhelo que cada día se acata menos: "Respetaré los secretos que se me confíen, incluso después de la muerte del paciente"; y

6. ¿No ha sucedido esto con Tatiana Tarasoff? ¿No lo estamos haciendo nosotros ahora porque, como permanecemos atrapados en las redes del derecho, nos referimos a ella por su nombre auténtico y no por un pseudónimo, según era costumbre en la tradición ética médica? Con el menoscabo de la confidencialidad ¿no estaremos abandonando "lo propio", Io eigen, según H eidegger?

\section{Los aspectos éticos del caso Tarasoff Dr. Fernando Novoa S.}

Coordinador Comisión de Ética SO NEPSYN

El caso presentado tiene el objetivo principal de motivarnos a reflexionar sobre los fundamentos que tiene el secreto profesional y cuales son sus límites ya que el aspecto central del caso fue el no haber dado a conocer a Tatiana la información obtenida por el psicólogo, en el marco de una relación clínica.

Esta es una materia de gran importancia, por la manera como se ejerce la Medicina en la actualidad. La información relativa a los pacientes tanto en clínicas y hospitales está expuesta a un gran número de personas que pudieran hacer un uso indebido de ella. Todas esas personas, no sólo los médicos, incluyendo los alumnos de M edicina, deben honrar en su conducta la obligación de respetar el secreto profesional.

El papel fundamental del profesional sanitario consiste en curar o atenuar los síntomas de la enfermedad. Para cumplir con estos objetivos, la sociedad otorga al profesional ciertos privilegios, como es el acceso a los aspectos más íntimos de la vida del paciente, tanto en lo que concierne a su persona física como psíquica. Incluso, durante la intervención el profesional puede tener acceso a información concerniente a la vida privada de otras personas que no le han consultado, pero que están vinculadas a su paciente. Toda esta información queda incluida en el secreto profesional. Este secreto deriva de la existencia del derecho a la intimidad que tienen todas las personas, y del hecho que el hombre es un ser social.

El hombre enfermo, debido al instinto natural de conservación, acude a consultar a otro hombre mejor preparado, para que le ayude a buscar la curación, el médico. Además entiende que esta ayuda va a ser más adecuada si confía plenamente en el profesional, y le entrega su intimidad. Esta es, en efecto, la única forma en que se puede ayudar al paciente. Desde los tiempos de Hipócrates se ha reconocido la obligación del médico de cautelar los secretos del paciente, debido a que para lograr que proporcione toda la información necesaria, es preciso que tenga la seguridad que sus secretos no serán revelados sin su consentimiento. Incluso, varios años después Percival en 1803 insistía "Los pacientes deben ser interrogados en un tono de voz que no pueda ser oído ni por azar".

Además, el secreto profesional tienefundamentos legales. La relación médico-paciente origina un contrato tácito que en el lenguaje jurídico se denomina cuasi-contrato ya que falta la expresión escrita. Se considera que en esta situación el deber del secreto profesional tiene como objetivo el bien común o de la sociedad, ya que su existencia crea un ambiente de pública confianza que 
facilita la apertura para que los pacientes den la información necesaria y en forma completa, hecho que facilita la posibilidad de que el médico ayude a su paciente. Por este motivo se estima que el secreto no sólo debe incluir la información directamente aportada por el paciente, sino que también todo aquello que el médico vea 0 conozca en el marco de la relación clínica, aún cuando el enfermo no hubiese querido que se hubiera producido ese conocimiento.

Sin embargo, este deber no es absoluto, y de hecho los códigos prohíben al médico la revelación de los secretos del paciente "a menos que sea necesario con el fin de proteger la salud o bienestar del paciente o de la comunidad". En esta situación es que se enmarca la conducta de Prosenjit Poddar y su psicólogo tratante Lawrence M oore.

Es ampliamente considerado un delito mantener en secreto una información que está vinculada con la posibilidad de que una persona esté en riesgo de sufrir un daño. En el caso que se comenta existía la información concreta de que había una real intención de Poddar de dar muerte a Tatiana. Esto debió haber sido puesto en conocimiento de ella, además de la policía. Se ha sostenido que incluso situaciones menos directas como la presentada, el médico que ha guardado el secreto puede ser demandado por los afectados. Tal sería el caso cuando se tiene conocimiento de que alguien está conduciendo vehículos, estando físicamente impedido de hacerlo. Si no se da la información necesaria para impedirlo y alguien resulta lesionado, el profesional puede ser demandado por negligencia. Lo que corresponde hacer es informar al paciente del riesgo que existe de conducir vehículos en su estado, y que debe dar cuenta a la autoridad. Además, comunicarle que en el caso de que continúe conduciendo, el mismo entregará la información al nivel que corresponda.

Como conclusión, se debe considerar una excepción tener que revelar a terceros la información que se ha obtenido en la relación médicopaciente. El secreto profesional es una condición necesaria para contar con toda la información requerida que va a permitir poder ayudar efectivamente a los pacientes.

Sin embargo, mantener el secreto no es obligatorio en un cierto número de circunstancias.

En el caso de Tatiana Tarasoff son tres las razones que justifican el no haber respetado el secreto profesional. La primera es el estado mental de Poddar que significaba un riesgo para Tatiana, por lo tanto ella debió ser informada de las intenciones de su pretendiente. La segunda se deriva de la anterior. Si Tatiana hubiera podido defenderse en mejor forma al estar debidamente informada del peligro que le acechaba, se podría haber protegido en forma más efectiva de Poddar, quien quizás no habría logrado cometer el crimen, lo que le habría favorecido al no haber sido imputado por semejante delito.

Finalmente, en tercer lugar tal como figura en el relato, la información clínica del paciente tiene que ser dada a conocer durante un proceso como respuesta a lo requerido por el juez debidamente acreditado. Pero, incluso en este caso, la información debe ser restringida exactamente a lo indispensable para que la justicia pueda cumplir con su labor, sin agregar otros aspectos de la vida del paciente, no relacionados a la materia del proceso. 